\title{
Unconventional etiology for heart failure and stroke: Non-compaction cardiomyopathy
}

\author{
Srikanth Seethala, Srinivasa V Jampana, Gur C Adhar, \\ Venkataraman Krishnaswami, Thomas Generalovich
}

\begin{abstract}
Introduction: Non-compaction cardiomyopathy is a rare, primary, genetic cardiomyopathy that results from intra-uterine arrest of the compaction process. It commonly involves the apical area of the left ventricle, and uncommonly involves the right ventricle. Case Report: A 45-year-old male presented to the hospital for heart failure. He was started on anticoagulation for the apical thrombus and later he suffered cerebrovascular accident. On careful evaluation he was found to have ventricular non-compaction both in right and left ventricles. Conclusion: Non-compaction cardiomyopathy should be considered in all refractory cases of heart failure. Anticoagulation is crucial in patients with decreased systolic function and atrial fibrillation.
\end{abstract}

Keywords: Biventricular non compaction, Ventricular non compaction, LVNC

Srikanth Seethala ${ }^{1}$, Srinivasa V Jampana ${ }^{2}$, Gur C Adhar ${ }^{3}$, Venkataraman Krishnaswami ${ }^{3}$, Thomas Generalovich ${ }^{3}$ Affiliations: ${ }^{1}$ Assistant Professor, Department of Internal Medicine, University of New Mexico, Albuquerque, NM, USA; ${ }^{2}$ Resident, Department of Internal Medicine, UPMCMercy hospital, Pittsburgh, PA; $3^{A}$ ssistant Professor, Department of Internal Medicine, UPMC-Mercy hospital, Pittsburgh, PA.

Corresponding Author: Srikanth Seethala, MSC10-555; 1 University of New Mexico, Albuquerque, New Mexico, 87131-0001, USA; Ph: 001-972-835-2873; Fax: 001-505277-9437; Email: srikanth.seethala@yahoo.com, sseethala@salud.unm.edu

Received: 16 August 2012

Accepted: 15 October 2012

Published: 01 February 2013
Seethala S, Jampana SV, Adhar GC, Krishnaswami V, Generalovich T. Unconventional etiology for heart failure and stroke: Non-compaction cardiomyopathy. International Journal of Case Reports and Images 2013;4(2):131-134.

$* * * * * * * * *$

doi:10.5348/ijcri-2013-02-278-CR-12

\section{INTRODUCTION}

Non-compaction cardiomyopathy is a rare, primary, genetic cardiomyopathy. Human cardiac morphogenesis commences with heart tube formation. Heart tube contains outer myoepicardial cells, middle acellular cardiac jelly (matrix of proteoglycans and glycosaminoglycans), and outer endocardial cells. During this time myocardial cells form deep trabeculae and intertrabecular recess so that blood percolates into intertrabecular recess in order to supply oxygen to myocardium. Compaction process, epicardial vessel formation and myocardial vascularization start around $5^{\text {th }}-8$ th week of gestation. Compaction proceeds from epicardial to endocardial surface and from the base to apex. Arrest of the cardiac compaction around this time will lead to non-compaction cardiomyopathy [1]. It commonly involves left ventricle. Right ventricular involvement is rare and here we are reporting a case of biventricular involvement.

\section{CASE REPORT}

A 45-year-old male presented to our institution for progressively worsening bilateral lower extremity edema, productive cough and generalized weakness that had been present for three weeks. His medical history was significant for recurrent bronchitis, smoking and a remote history of cocaine abuse. His family history was unremarkable for any cardiac disease. On physical 
examination, blood pressure was 142/90 $\mathrm{mmHg}$, and heart rate was 109/min.

The patient had marked jugular vein distention, S3 gallop, bilateral lower extremity edema and normal pulmonary examination. The electrocardiogram revealed sinus tachycardia with voltage criteria consistant with left ventricular hypertrophy and repolarization abnormalities (Figure 1). Blood chemistry revealed a serum creatinine of $1.8 \mathrm{mg} / \mathrm{dl}$, blood urea nitrogen of $24 \mathrm{mg} / \mathrm{dl}$, and mild proteinuria. Chest X-ray revealed cardiomegaly and probable left upper lobe infiltrate. A 2Dechocardiogram was performed which revealed moderate left ventricular dilatation, severely reduced left ventricular systolic function (estimated ejection fraction of 10-20\%) and a large mobile apical thrombus. He was treated with heparin, warfarin, lisnopril, carvedilol, diuretics and digoxin. After achieving therapeutic anticoagulation and a good control of symptoms of heart failure he was discharged with a diagnosis of non-ischemic cardiomyopathy with a referral to a warfarin clinic for anticoagulation monitoring.

He presented to the emergency department 10 days after discharge for worsening of bilateral lower extremity edema. On repeat laboratory work up, his serum creatinine had decreased to $1.19 \mathrm{mg} / \mathrm{dL}$, PT was 14.2 and INR was 1.2. He was admitted to the hospital for worsening heart failure. Next morning, he was found to be unresponsive. A computed tomography (CT) scan of the head failed to reveal any hemorrhage and magnetic resonance imaging (MRI) of brain revealed a hyper acute embolic cerebrovascular accident (CVA) in the left middle and anterior cerebral artery territory. An interventional neurologist performed thrombectomy and vasodilator therapy. We were consulted for apical thrombus management. We reviewed previous 2Dechocardiogram and suspected non-compaction cardiomyopathy because of hypertrabeculations and a spongy appearance of the myocardium.

A transesophageal echocardiogram (TEE) was performed to confirm the diagnosis and for detailed evaluation of the apical thrombus. TEE revealed biventricular non-compaction with marked hypertrabeculations in left and right ventricles and a ratio of the non-compacted to compacted myocardium greater than 2 (Figure 2) [1]. A left ventricular mural thrombus could not be either confirmed or excluded due to large mobile trabeculations. Unfortunately, his postprocedural course was complicated by the development of cerebral edema and a midline shift. He underwent decompressive hemicraniotomy and later suffered multi-system organ failure and was withdrawn from life support at his family's request.

An autopsy confirmed hypertrabeculations of both right and left ventricular myocardium, and a mural thrombus (Figure 3).

\section{DISCUSSION}

Ventricular non-compaction cardiomyopathy (VNC) is a rare, primary, genetic cardiomyopathy characterized

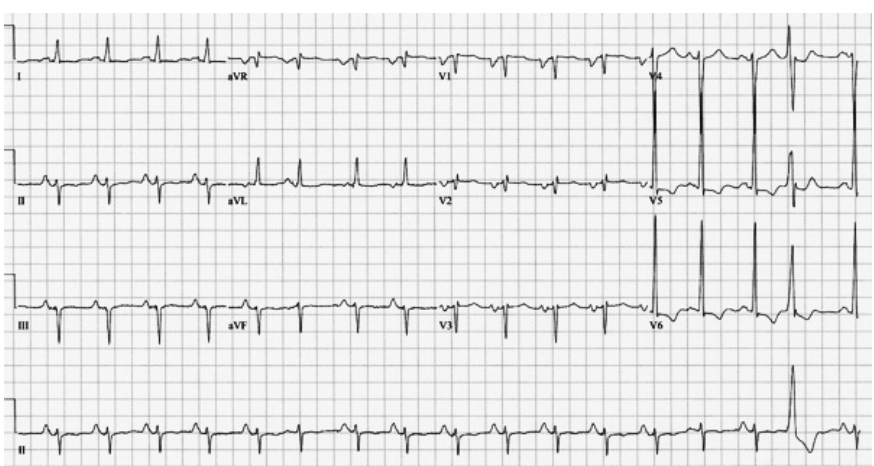

Figure 1: Sinus tachycardia with voltage criteria consistant with left ventricular hypertrophy and repolarization abnormalities.

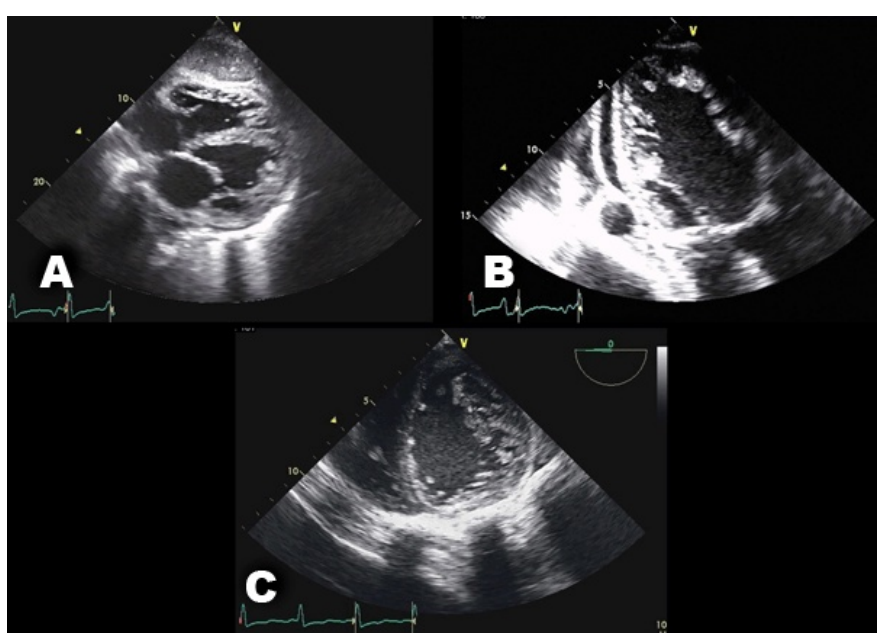

Figure 2: (A) Four chamber sub costal view showing hypertrabeculations, (B) Apical three chamber view showing hypertrabeculations, (C) Gastric short axis view of TEE showing hypertrabeculations.

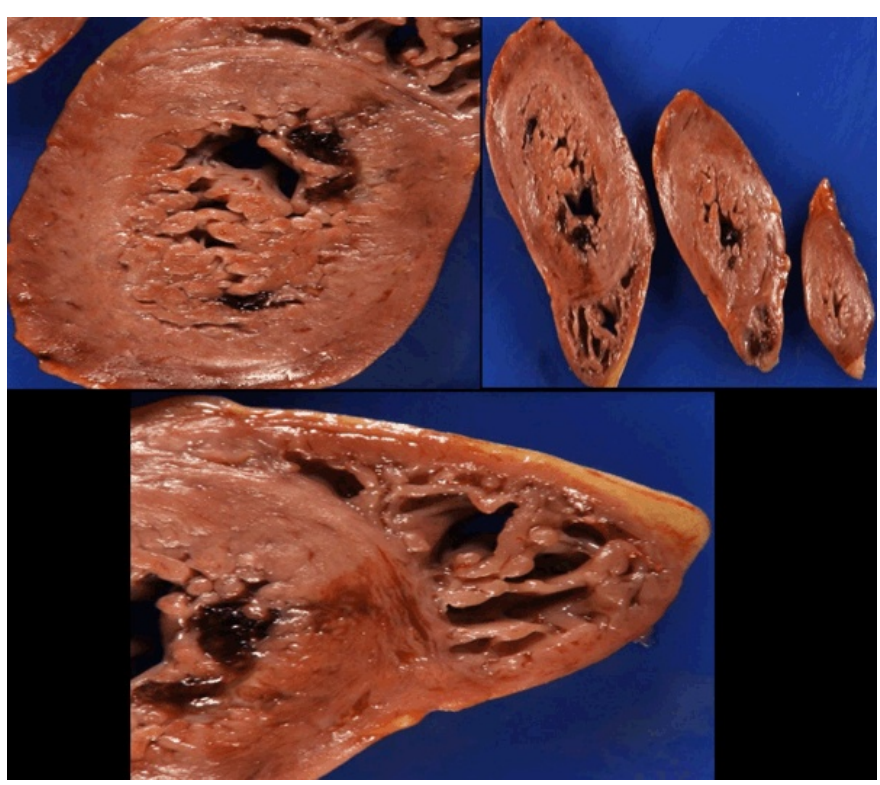

Figure 3: Autopsy findings of right and left ventricular hypertrabeculations, and a mural thrombus. 
by hypertrabeculations and deep intertrabecular recesses. VNC is secondary to the intra-uterine arrest of the compaction process which occurs during the $5^{\text {th-8th }}$ week of gestation. The prevalence of VNC is reported to be between $0.06-0.24 \%$ [2]. VNC commonly involves the apical, mid-lateral, inferior, anterior and septal areas of left ventricle, leading to the name left ventricular non compaction (LVNC). Right ventricular involvement is uncommon $[1,2]$.

The clinical presentation of LVNC is widely variable and ranges from incidental diagnosis to sudden cardiac death. The most common presentations of VNC are heart failure, arrhythmias and thromboembolic phenomenon respectively $[1,2]$.

Systolic dysfunction is the primary contributor of heart failure. Even though the exact etiology for systolic dysfunction is not clear, it is postulated that it could be secondary to subendocardial hypoperfusion, and microcirculatory dysfunction. Abnormalities of diastolic function are common, ranging from abnormal ventricular relaxation to restrictive cardiomyopathy $[3,4]$.

Some studies have shown a correlation between the number of non-compacted segments and the severity of LV dysfunction [5, 6]. In contrast, one relatively large study failed to support the relation between number of non-compacted segments and the global systolic function [3]. It is difficult to compare these studies, as the imaging modalities, LVNC diagnostic criteria, and the average left ventricular ejection fraction (LVEF) differed in these studies.

The prevalence of atrial fibrillation in LVNC is reported as high as 26\% [7]. Along with ventricular dysfunction, atrial fibrillation can worsen heart failure because of the loss of atrial kick and dyssynchrony between atrial and ventricular contraction. In addition to atrial fibrillation, left bundle branch block (LBBB) has been described in between $15-44 \%$ of the patients $[1,7]$. Generally, patients with LBBB have lower ejection fractions when compared to their counterparts. LBBB can also be a contributor to lower ejection fraction and heart failure in (LVNC) patients.

In pediatric patients, a reduced early diastolic velocity at the lateral mitral annulus appears to be a more sensitive predictor for heart failure hospital admission when compared to the LVEF [8]. At this time, we are not sure whether or not we can extrapolate the study results to adult population as the disease spectrum is slightly different in pediatric patients when compared to adults. Even in adults, patients with a higher LVEF tend to be younger [1]. It is critical to identify LVNC and optimize medical management early in the course, since elevated left ventricular filling pressures and hospitalization for heart failure are independently associated with high mortality and the need for cardiac transplantation [1].

Thromboembolic events are common in LVNC. Deep intertrabecular recesses are presumed to be the nidus for thrombus formation [9]. It may be difficult to identify small mural thrombi in LVNC due to the presence of large, mobile trabeculations. In our case, while the initial transthoracic echocardiogram identified a left ventricular apical thrombus, we were not able to visualize a clear thrombus on trans-esophageal echocardiogram. The final autopsy revealed a small mural thrombus.

Left ventricular non-compaction by itself may not be an independent risk factor for thromboembolic events and the increased risk may be related to severe systolic dysfunction. All LVNC patients with systolic dysfunction should be anticoagulated [9, 10]. Patients with associated atrial fibrillation are at high risk for having peripheral embolic events and should be anticoagulated irrespective of their CHADS2 score index.

It is critical not only to initiate warfarin but also to maintain the INR in therapeutic range. Newer oral anticoagulation agents may reduce the likelihood of subtherapeutic anticoagulation, and have proven to be effective in patients with non-valvular atrial fibrillation. These agents could potentially be used in the management of atrial fibrillation in patients with LVNC, but their efficacy in the prevention of left ventricular mural thrombi is still unproven.

\section{CONCLUSION}

Ventricular non-compaction cardiomyopathy should be considered in the differential diagnosis of refractory heart failure.

$* * * * * * * * *$

\section{Author Contributions}

Srikanth Seethala - Substantial contributions to conception and design, Acquisition of data, Analysis and interpretation of data, Drafting the article, Revising it critically for important intellectual content, Final approval of the version to be published

Srinivasa V Jampana - Substantial contributions to conception and design, Acquisition of data, Analysis and interpretation of data, Drafting the article, Revising it critically for important intellectual content, Final approval of the version to be published

Gur C Adhar - Substantial contributions to conception and design, Acquisition of data, Analysis and interpretation of data, Drafting the article, Revising it critically for important intellectual content, Final approval of the version to be published

Venkataraman Krishnaswami - Substantial contributions to conception and design, Acquisition of data, Analysis and interpretation of data, Drafting the article, Revising it critically for important intellectual content, Final approval of the version to be published Thomas Generalovich - Substantial contributions to conception and design, Acquisition of data, Analysis and interpretation of data, Drafting the article, Revising it critically for important intellectual content, Final approval of the version to be published

\section{Guarantor}

The corresponding author is the guarantor of submission. 


\section{Conflict of Interest}

Authors declare no conflict of interest.

\section{Copyright}

(C) Srikanth Seethala et al. 2013; This article is distributed under the terms of Creative Commons Attribution 3.0 License which permits unrestricted use, distribution and reproduction in any means provided the original authors and original publisher are properly credited. (Please see www.ijcasereportsandimages.com /copyright-policy.php for more information.)

\section{REFERENCES}

1. Habib G, Charron P, Eicher JC, et al. Isolated left ventricular non-compaction in adults: clinical and echocardiographic features in 105 patients. Results from a French registry. Eur J Heart Fail 2011;13(2):177-85.

2. Captur G, Nihoyannopoulos P. Left ventricular noncompaction: genetic heterogeneity, diagnosis and clinical course. Int J Cardiol 2010;140(2):145-3.

3. Fazio G, Corrado G, Novo G, et al. Ventricular dysfunction and number of non compacted segments in non compaction: non-independent predictors. Int J Cardiol 2010;141(3):250-3.

4. Ichida F, Hamamichi Y, Miyawaki T, et al. Clinical features of isolated noncompaction of the ventricular myocardium: long-term clinical course, hemodynamic properties, and genetic background. J Am Coll Cardiol 1999;34(1):233-40.
5. Dellegrottaglie S, Pedrotti P, Roghi A, Pedretti S, Chiariello M, Perrone-Filardi P. Regional and global ventricular systolic function in isolated ventricular non-compaction Pathophysiological insights from magnetic resonance imaging. Int J Cardiol. $2012 \mathrm{Jul}$ 26;158(3):394-9.

6. Lofiego C, Biagini E, Ferlito M, et al. Paradoxical contributions of non-compacted and compacted segments to global left ventricular dysfunction in isolated left ventricular noncompaction. Am J Cardiol 2006;97(5):738-41.

7. Oechslin EN, Attenhofer Jost CH, Rojas JR, Kaufmann PA, Jenni R. Long-term follow-up of 34 adults with isolated left ventricular noncompaction: a distinct cardiomyopathy with poor prognosis. J Am Coll Cardiol 2000;36(2):493-500.

8. McMahon CJ, Pignatelli RH, Nagueh SF, et al. Left ventricular non-compaction cardiomyopathy in children: characterisation of clinical status using tissue Doppler-derived indices of left ventricular diastolic relaxation. Heart 2007;93(6):676-81.

9. Pitta S, Thatai D, Afonso L. Thromboembolic complications of left ventricular noncompaction: case report and brief review of the literature. J Clin Ultrasound 2007;35(8):465-8.

10. Stöllberger C, Finsterer J. Left ventricular hypertrabeculation/noncompaction and stroke or embolism. Cardiology 2005;103(2):68-72.
Access full text article on other devices

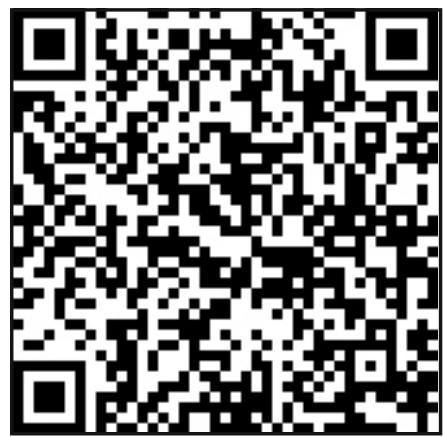

Access PDF of article on other devices

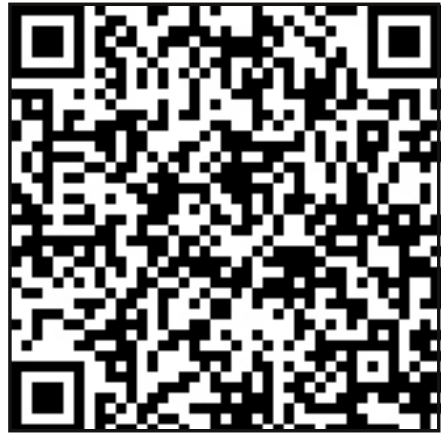

